\title{
LXRa promotes cell metastasis by regulating the NLRP3 inflammasome in renal cell carcinoma
}

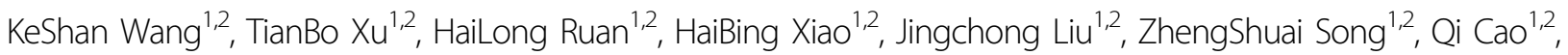 \\ Lin Bao ${ }^{1,2}$, Di Liu' ${ }^{1,2}$, Cheng Wang ${ }^{1,2}$, Gong Cheng ${ }^{1,2}$, HuaGeng Liang ${ }^{1,2}$, ZhaoHui Chen ${ }^{1,2}$, HongMei Yang ${ }^{3}$, \\ Ke Chen ${ }^{1,2}$ and XiaoPing Zhang ${ }^{1,2}$
}

\begin{abstract}
Notwithstanding the researches on biomarkers and targeted therapies in renal cell carcinomas (RCC) have made progress in the last decades, the application of the biomarkers and targeted therapy agents for RCC in clinic are restricted because of their limitation or side effects. Liver X receptors (LXRs) and the NLRP3 inflammasome have been the research hotspots in recent years. In our study, we integrated bioinformatics analysis, molecular biology experiments and biological function experiments to study the roles of LXRa and the NLRP3 inflammasome in RCC. The study demonstrated that the elevated LXRa expression is correlated with a poor prognosis in RCC. Furthermore, our study revealed the expression levels and roles of the NLRP3 inflammasome in RCC for the first time. This research demonstrated that LXRa could promote the metastasis of RCC cells by suppressing the expression of the NLRP3 inflammasome. In Brief, LXRa had the possibility to be a novel diagnostic and prognostic biomarker and therapeutic target in renal cell cancer and LXRa could regulate the metastasis of renal cell cancer via NLRP3 inflammamsome.
\end{abstract}

\section{Introduction}

Renal cell carcinoma (RCC), characterized by high incidence and high mortality rates, constitutes $3 \%$ of adult malignancies. It is estimated that 65,340 new cases of RCC will be diagnosed and that 14,970 patients will die from RCC in 2018 in the USA. ${ }^{1}$. Clear-cell renal cell carcinoma (ccRCC) accounts for $75 \%$ of renal cell carcinomas and is the most familiar subtype of $\mathrm{RCC}^{2}$. According to reports, $20-30 \%$ RCC patients present with local or distant metastasis at the time of diagnosis, and the response to chemotherapy or radiotherapy in the majority of the

\footnotetext{
Correspondence: Ke Chen (shenke@hust.edu.cn) or XiaoPing Zhang (xzhang@hust.edu.cn)

'Department of Urology, Union Hospital, Tongji Medical College, Huazhong University of Science and Technology, Wuhan 430022, China

${ }^{2}$ Institute of Urology, Union Hospital, Tongji Medical College, Huazhong University of Science and Technology, Wuhan 430022, China

Full list of author information is available at the end of the article.

These authors contributed equally: KeShan Wang, TianBo Xu

Edited by A. Stephanou
}

advanced RCC patients is dissatisfying ${ }^{3}$. We considered that the exploration and application of effective diagnostic and prognostic biomarkers for RCC patients could be useful for ccRCC diagnosis and therapy. Although the occurrence of target drugs for RCC have improved therapeutic outcomes of advanced and metastatic RCC, poor response and high incidence of side effects for these agents restrict their clinical application ${ }^{4}$. Therefore, it is urgent to explore new potential biomarkers and therapeutic targets for RCC.

As a kind of nuclear receptor superfamily, the liver $\mathrm{X}$ receptor (LXR) family, including LXR $\alpha$ (LXRA, NR1H3) and LXR $\beta$ (LXRB, NR1H2), is an important regulator of several kinds of cancers (prostate cancer, breast cancer, etc.). While LXR $\beta$ is expressed widely throughout various tissues and organs, LXR $\alpha$ is mainly expressed in liver, kidney, spleen and intestine ${ }^{5-8}$. According to the studies, LXRs could influence the inflammatory response via regulating the expression of inflammatory cytokines,

\section{(c) The Author(s) 2019}

(c) (i) Open Access This article is licensed under a Creative Commons Attribution 4.0 International License, which permits use, sharing, adaptation, distribution and reproduction cc) in any medium or format, as long as you give appropriate credit to the original author(s) and the source, provide a link to the Creative Commons license, and indicate if changes were made. The images or other third party material in this article are included in the article's Creative Commons license, unless indicated otherwise in a credit line to the material. If material is not included in the article's Creative Commons license and your intended use is not permitted by statutory regulation or exceeds the permitted use, you will need to obtain permission directly from the copyright holder. To view a copy of this license, visit http://creativecommons.org/licenses/by/4.0/. 
including STAT, IL-18, TNF- $\alpha$ and so on ${ }^{9-11}$. The inflammasome, a novel research focus, is a kind of cytosolic multi-protein complex which could participate in the development of several cancers via regulation of tumor inflammation and immunity ${ }^{12,13}$. The NLRP3 inflammasome, one of the most critical inflammasomes, contains NLRP3, apoptosis-associated speck-like protein containing a CARD (ASC), and caspase-1 (CASP1). The NLRP3 inflammasome functioned as a platform for caspase-1 activation and the activated caspase- 1 could cleave the pro-IL-1 $\beta$ into mature IL- $1 \beta^{14}$. According to the researches, the NLRP3 inflammasome could regulate the development of the cancers. Activating of the NLRP3 inflammasome was correlated with the azoxymethaneinduced colorectal cancer ${ }^{15}$. In lung cancer, the NLRP3 inflammasome could enhance the cell proliferation and migration and was a potential therapy target for lung cancer $^{16}$. Knockdown of NLRP3 could inhibit the proliferation and invasion of pancreatic cancer cells ${ }^{17}$. Activation of the NLRP3 inflammasome could promote the carcinogenesis in squamous cell carcinoma of the head and neck ${ }^{18}$.

The expression and biological functions of LXRs and the NLRP3 inflammasome, as well as their possible correlation to RCC, is still unclear. Based on the above studies, firstly, we explored the expression level and function of LXR $\alpha$ in RCC. Our study indicated that LXR $\alpha$ could be a biomarker for diagnosis and prognosis in ccRCC. Moreover, we investigated the mechanism of LXR $\alpha$ regulating the development of RCC and the study revealed that LXR $\alpha$ could regulate the metastasis of ccRCC via the NLRP3 inflammasome for the first time.

\section{Results}

\section{Elevated LXRa expression correlates with clinical features} in cCRCC

Although bioinformatics analysis of LXR (LXR $\alpha$ and LXR $\beta$ ) expression in ccRCC indicated that both LXR $\alpha$ and LXR $\beta$ mRNA expression in ccRCC cancer tissue was higher than that in corresponding adjacent normal tissues, the elevated degree of LXR $\alpha$ expression in ccRCC cancer tissues compared with normal kidney were much higher than those of LXR $\beta$ (Fig. 1a, Fig. S1A). Further bioinformatics analysis of LXR $\alpha$ in ccRCC indicated that its expression levels were related with clinicopathological features in ccRCC patients (Table 1, Fig. 1b). KaplanMeier curves indicated that high expression of LXR $\alpha$ or LXR $\beta$ predicted a poor prognosis in ccRCC patients, and the overexpression of LXR $\alpha$ in ccRCC patients who were $\mathrm{T} 1+\mathrm{T} 2$ stage $(p=0.0105)$ or $\mathrm{G} 1+\mathrm{G} 2$ stage $(p=0.0146)$ was also associated with a poor prognosis (Fig. 1c, Fig. S1B). Additionally, LXR $\alpha$ effectively differentiated ccRCC from paracancerous normal tissues with an area under the curve (AUC) of 0.8829 (95\% CI: 0.8241-0.9418; $p<0.0001$ ), and LXR $\alpha$ expression may be a potential diagnostic biomarker for ccRCC patients with (T1+T2) VS (T3+T4) stage and $(\mathrm{G} 1+\mathrm{G} 2)$ VS $(\mathrm{G} 3+\mathrm{G} 4)$ stage according to the results of ROC curves (Fig. 1d). To verify LXR $\alpha$ expression levels in ccRCC cell lines and tissues, we extracted the RNA and protein from both ccRCC cells and tissues for qRT-PCR assay and western blotting analysis, respectively. Our results indicated that the protein and mRNA expressions of LXR $\alpha$ were up-regulated in both ccRCC cells and tissues (Figs. 1e, f). Simultaneously, results of IHC analysis of LXR $\alpha$ also revealed the same result (Fig. 1g). In addition, the qRT-PCR analysis of LXR $\beta$ demonstrated that the degree of elevations of LXR $\beta$ expression in ccRCC cancer tissues were lower than LXR $\alpha$ expression (Fig. S1C). These findings revealed that the expression of LXR $\alpha$ is elevated in ccRCC cancer tissues and that the expression of LXR $\alpha$ was correlated with the clinical features of ccRCC and could be a potential biomarker for ccRCC diagnosis and prognosis.

\section{LXRa regulates cell migration and invasion in cCRCC cells}

According to the results above, we predicted that both LXR $\alpha$ and LXR $\beta$ might be oncogenes in ccRCC. However, elevation degree of LXR $\alpha$ in ccRCC cancer tissue compared to normal kidney tissues was much higher than that of LXR $\beta$. Furthermore, reports have also revealed that expression of LXR $\alpha$ is mainly in lipid metabolism-related tissues (kidney, liver, etc.), while LXR $\beta$ is widely expressed across tissues. Thus, we considered that LXR $\alpha$ expression in renal cancer may be more specific and functional than LXR $\beta$ and we decided to focus on the roles of LXR $\alpha$ in ccRCC in our study. To verify the function of LXR $\alpha$ in ccRCC, we utilized ACHN and 786-O cells with stable knockdown of LXR $\alpha$ and ACHN and A498 cells with stable overexpression of LXR $\alpha$. Cell transfection efficiency and induction of constructs were verified via western blotting and qRT-PCR (Figs. 2a, b). Furthermore, our results indicated that expression of LXR $\alpha$ in ccRCC was mainly correlated with aggressiveness in ccRCC. In transwell assays, 786-O and ACHN normal control cells possessed greater migration and invasion capacity than corresponding LXR $\alpha$ stable knockdown cells. Congruously, cell migration and invasion in A498 and ACHN cells with stable overexpression of LXR $\alpha$ were faster compared to corresponding normal control cell lines (Fig. 2c). Similarly, wound healing assays revealed that the knockdown of LXR $\alpha$ could suppress the scratch healing capacity of ccRCC, while the overexpression of LXR $\alpha$ could enhance healing ability (Fig. 2d). In addition to transwell assays, apoptosis and cell cycle assays revealed that knockdown of LXR $\alpha$ had almost no effect on these processes in ccRCC cells (Fig. S2A, B). Collectively, these results demonstrated that LXR $\alpha$ expression mostly enhanced the cell aggressiveness ability of ccRCC cells. 


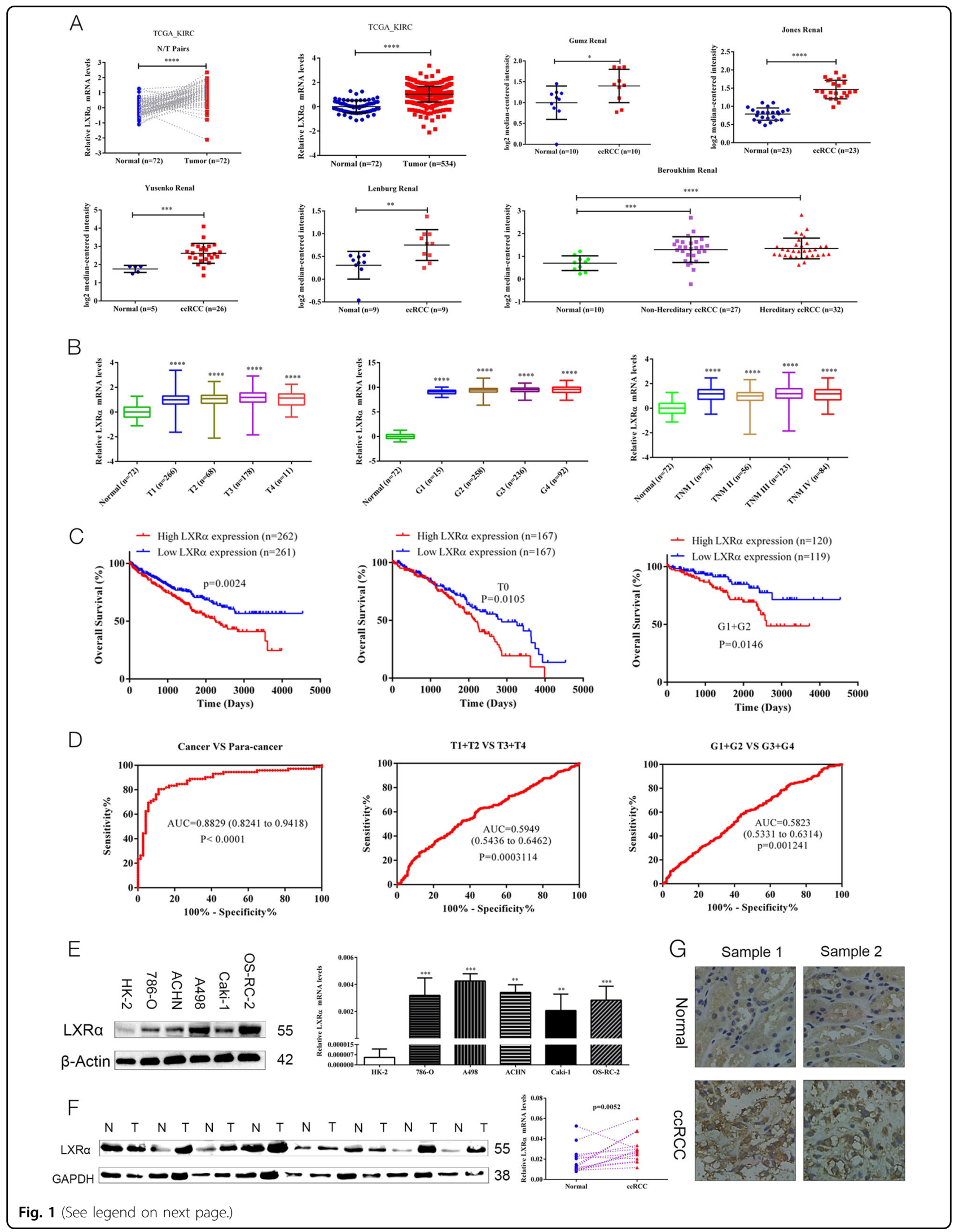


(see figure on previous page)

Fig. 1 LXRa expression is elevated in ccRCC and correlates with clinical features. a mRNA levels for LXRa were obtained from TCGA_KIRC datasets and Oncomine datasets (Gumz Renal, Jones Renal, Yusenko Renal, Lenburg Renal, and Beroukhim Renal). b mRNA levels of LXRa compared with different clinicopathological features: T stage, G stage and TNM stage. c The OS of ccRCC patients is correlated with expression of LXRa, and high expression of $L X R a$ indicates a poor prognosis in $T 0$ and $G 1+G 2$ stage $c c R C C$ patients. $\mathbf{d}$ Expression of $L X$ Ra differentiates the adjacent normal tissues and $c C R C C$ cancer tissues according to the ROC curve analysis. ROC curve analysis of LXRa expression in subgroups of ccRCC patients with (T1+T2) VS (T3+T4) stage and (G1+G2) VS (G3+G4) stage was also performed. e Western blotting and qRT-PCR analysis of LXRa protein and mRNA expression levels in ccRCC cell lines and HK-2 cells. $\mathbf{f}$ Expression levels of LXRa protein and mRNA in cCRCC cancer tissue and normal kidney tissue. $\mathbf{g}$ Immunohistochemistry (IHC) analysis of LXRa expression in CCRCC tissues and normal kidney tissues. Original magnification x200 (Data are shown as mean \pm SD. ${ }^{* * *} P<0.0001 ;{ }^{* *} P<0.001 ;{ }^{* *} P<0.01 ;{ }^{*} P<0.05$, and ns means no significant difference compared with the corresponding control)

Table 1 Correlation between LXRa mRNA expression and clinicopathological parameters of KIRC

\begin{tabular}{|c|c|c|c|c|}
\hline \multirow[t]{2}{*}{ Parameter } & \multirow[t]{2}{*}{ Number } & \multicolumn{2}{|c|}{$\begin{array}{l}\text { LXRa mRNA } \\
\text { expression }\end{array}$} & \multirow[t]{2}{*}{$P$ value } \\
\hline & & $\begin{array}{l}\text { Low } \\
(n=261)\end{array}$ & $\begin{array}{l}\text { High } \\
(n=262)\end{array}$ & \\
\hline \multicolumn{5}{|l|}{ Age (years) } \\
\hline$<60$ & 260 & 144 & 116 & 0.013 \\
\hline$>=60$ & 263 & 117 & 146 & \\
\hline \multicolumn{5}{|l|}{ T stage } \\
\hline $\mathrm{T} 1+\mathrm{T} 2$ & 334 & 187 & 147 & 0.000 \\
\hline $\mathrm{T} 3+\mathrm{T} 4$ & 189 & 74 & 115 & \\
\hline \multicolumn{5}{|l|}{$N$ stage } \\
\hline No & 507 & 254 & 253 & 0.617 \\
\hline $\mathrm{N} 1$ & 16 & 7 & 9 & \\
\hline \multicolumn{5}{|l|}{ M stage } \\
\hline Mo & 445 & 229 & 216 & 0.089 \\
\hline M1 & 78 & 32 & 46 & \\
\hline \multicolumn{5}{|c|}{$\begin{array}{l}\text { Neoplasm } \\
\text { histologic grade }\end{array}$} \\
\hline $\mathrm{G} 1+\mathrm{G} 2$ & 244 & 140 & 104 & 0.001 \\
\hline $\mathrm{G} 3+\mathrm{G} 4$ & 279 & 121 & 158 & \\
\hline \multicolumn{5}{|c|}{ Pathologic stage } \\
\hline $1+2$ & 316 & 181 & 135 & 0.000 \\
\hline $3+4$ & 207 & 80 & 127 & \\
\hline \multicolumn{5}{|l|}{ Gender } \\
\hline Male & 341 & 157 & 184 & 0.016 \\
\hline Female & 182 & 104 & 78 & \\
\hline \multicolumn{5}{|l|}{ Laterality } \\
\hline Left & 246 & 116 & 130 & 0.236 \\
\hline Right & 277 & 145 & 132 & \\
\hline
\end{tabular}

Significance of bold values are $p<0.05$
LXRa could negative regulate the expression level of the NLRP3 inflammasome in ccRCC cells

After we confirmed the biological function of LXR $\alpha$ in ccRCC, we explored the possible mechanisms enhancing tumor growth, migration and invasion of LXR $\alpha$ in ccRCC cells. As we mentioned before, LXR $\alpha$ could regulate the expression of typical inflammatory cytokines. However, the relationship between $L X R \alpha$, the NLRP3 inflammasome, and the biological effects of their correlation in ccRCC have not been reported. To investigate these problems, we evaluated the expression of NLRP3 in ccRCC cancer tissues. Our study revealed that the expressions of NLRP3 in ccRCC cancer tissues were significant lower than that in normal kidney tissues for the first time (Fig. S2C). According to the western blotting results, knockdown of $\mathrm{LXR} \alpha$ could up-regulate the expression of NLRP3 in ccRCC cells, while LXR $\alpha$ overexpression could decrease the expression level of NLRP3 compared to corresponding normal control cells (Fig. 3a). Once the NLRP3 inflammasome is activated by LPS, the expression and secretion of cleaved caspase-1 (CASP1) and IL- $1 \beta$ is enhanced ${ }^{19}$. In addition, the biological activity and function of their cleavage forms are stronger than their precursor forms. Thus, in order to verify that the expression of LXR $\alpha$ regulates the expression and activation of the NLRP3 inflammasome, we measured the expression levels of NLRP3, CASP1 and IL-1 $\beta$ in ccRCC cells with stable knockdown of LXR $\alpha$ (Figs. 3b, c). In accordance with expression level of NLRP3, knockdown of LXR $\alpha$ resulted in the up-regulation of CASP1 and IL$1 \beta$ expression and enhanced their cleavage products, CASP1 (p20) and IL-1 $\beta$ (p15), compared with pro-CASP1 and pro-IL-1 $\beta$. Meanwhile, the expression level of the NLRP3 inflammasome was dose-dependent of LPS (Fig. 3b). To prove expression of CASP1 and IL-1 $\beta$ was NLRP3-dependent, we transfected si-NLRP3 into ccRCC cells with stable LXR $\alpha$-knockdown. In cells with stable LXR $\alpha$-knockdown, si-NLRP3 transfection inhibited the up-regulation of CASP1 and IL-1 $\beta$ that was caused by LXR $\alpha$-knockdown (Figs. 3d, e). Thus, we declared that the 


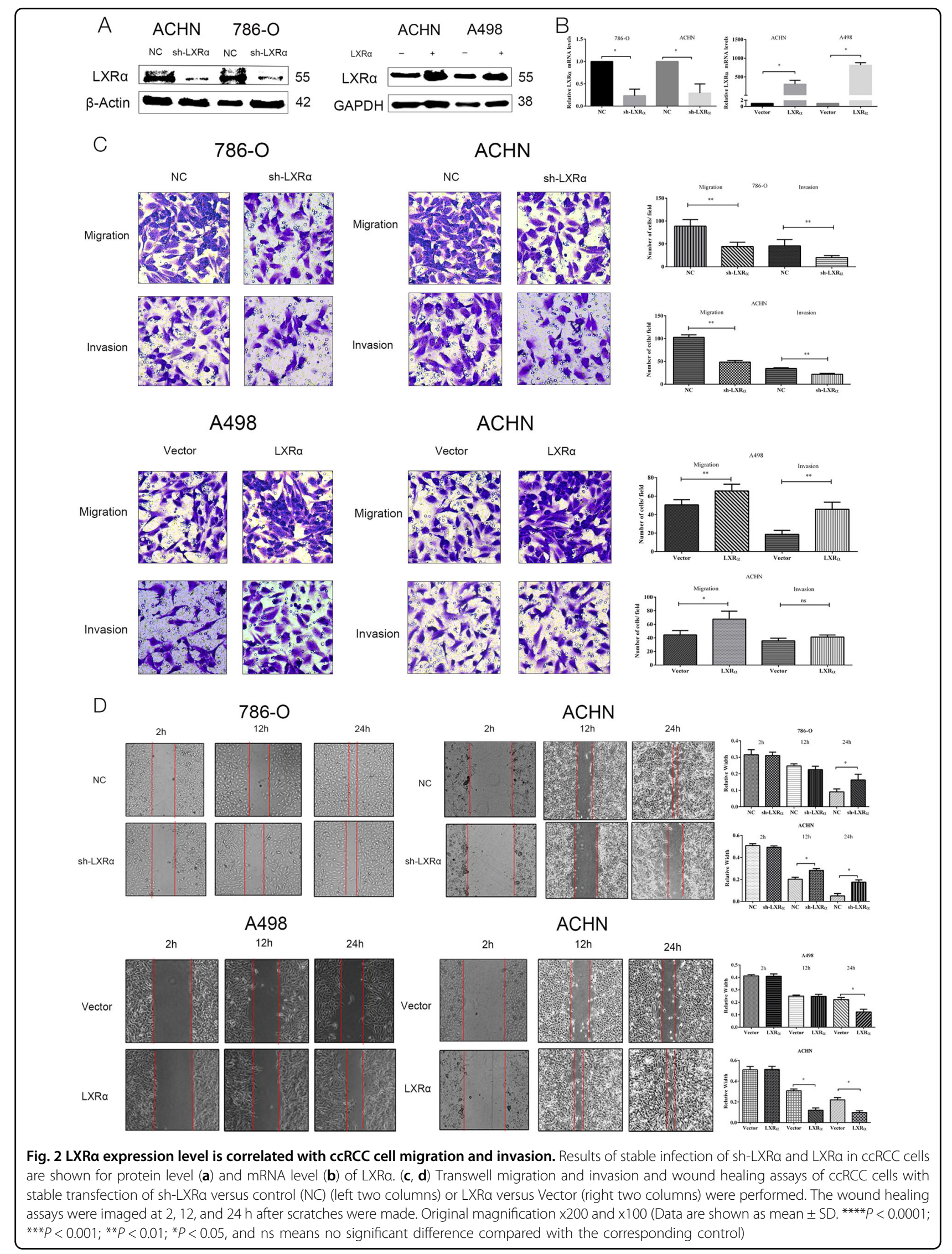




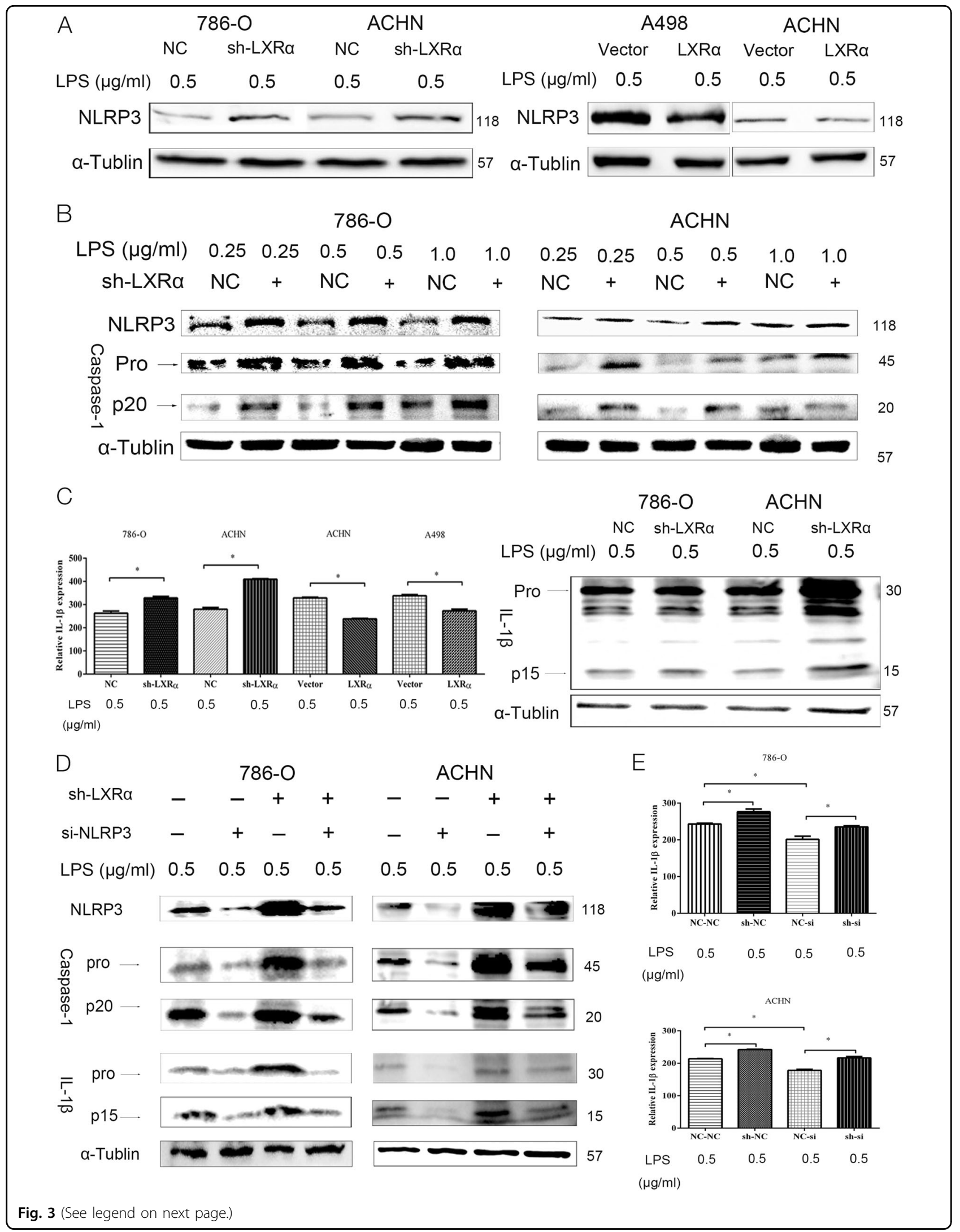


(see figure on previous page)

Fig. 3 NLRP3 inflammasome expression is negatively regulated by LXRa in ccRCC cells. a Expression levels of NLRP3 were elevated in 786-O and ACHN cells with stable LXRa knockdown. NLRP3 expression was down-regulated in A498 and ACHN cells with LXRa overexpression. $\mathbf{b}$ Expression levels of NLRP3, pro-CASP1, and cleaved CASP1 (p20) in 786-O and ACHN cells with stable LXRa knockdown. LPS stimulus was performed at 0.25, 0.5, and $1.0 \mathrm{\mu g} / \mathrm{ml}$. c ELISA assays were used to analyze exocrine IL-1 $\beta$ expression levels in culture supernatant from ccRCC cells with stable LXRa knockdown or LXRa overexpression. Protein expression of pro-IL-1 $\beta$ and cleaved IL-1 $(p 15)$ in 786-O and ACHN cells with stable LXRa knockdown were detected by western blotting. $\mathbf{d}$ Transient transfection of si-NLRP3 inhibited induction of pro-CASP1, cleaved CASP1 (p20), pro-IL-1 $\beta$ and cleaved IL-1 $\beta$ protein expression, which were elevated in stable LXRa knockdown cells. e Elevation of exocrine IL-1 $\beta$ expression in 786-O and ACHN cells with stable LXRa knockdown was blocked by transient transfection with si-NLRP3. NC-NC, sh-NC, NC-si, and sh-si indicates NC+NC, sh-LXRa+NC, NC+siNLRP3, and sh-LXRa+si-NLRP3, respectively. (Data are shown as mean \pm SD. ${ }^{* * *} P<0.0001$; ${ }^{* * *} P<0.001 ;{ }^{* *} P<0.01 ;{ }^{*} P<0.05$, and ns means no significant difference compared with the corresponding control)

expression of NLRP3 in ccRCC cancer tissues was downregulated and the expression of LXR $\alpha$ could dampen expression of the NLRP3 inflammasome in ccRCC cells.

\section{Knockdown of NLRP3 reverses the altered biological function caused by LXRa in ccRCC cells}

The above results indicate that the NLRP3 inflammasome might be a possible downstream target of LXR $\alpha$. To examine whether the knockdown of NLRP3 could interrupt the inhibitory effects on aggressiveness caused by LXR $\alpha$ knockdown in ccRCC cells, we performed rescue experiments by co-transfecting with sh-LXR $\alpha$ lentivirus (vs. the negative control) and si-NLRP3 (vs. the negative control) into ACHN and 786-O cells. Similar to the results shown above, transient transfection of si-NLRP3 inhibited the up-regulation of NLRP3, CASP1 and IL-1 $\beta$ caused by LXR $\alpha$-knockdown. The wound healing assays verified that the weakening effect in cell migration induced by LXR $\alpha$ knockdown was effectively reversed by NLRP3 knockdown (Fig. 4a). Besides, according to the results of transwell migration and invasion assays in 786-O and ACHN that were cotransfection with sh-LXR $\alpha$ (vs. the negative control) and si-NLRP3 (vs. the negative control), we confirmed that knockdown of NLRP3 could reverse the inhibitory effect in ccRCC cell metastasis caused by knockdown of LXR $\alpha$ (Fig. 4b). It was notable that knockdown of NLRP3 could promote the cell migration and invasion in ccRCC according to our wound healing, transwell migration and invasion assays. Based on above results, we considered that the NLRP3 was tumor suppressor gene in ccRCC and the NLRP3 inflammasome is an important functional effector of LXR $\alpha$ in ccRCC cells for the first time.

\section{LXRa knockdown suppressed metastasis of ccRCC cells in vivo}

To explore the roles of LXR $\alpha$ in ccRCC in vivo, 786-O cells with stable knockdown of LXR $\alpha$ versus normal control (NC) were injected into the tail vein of nude mice. Mice were euthanized 28 days after injection, and the liver, lung, spleen and kidney were isolated and analyzed. After injection of 786-O cells, the fold change of mouse body weight in the LXR $\alpha$ knockdown group was significant higher than in the NC group, and the number of macroscopic metastases on the surface of the liver in the NC group was much more than that in the LXR $\alpha$ knockdown group (Fig. 5a). Mean fluorescence intensity values for the liver, spleen and lung in the $\mathrm{NC}$ group were obviously higher than the values in corresponding organs of LXR $\alpha$ knockdown-injected mice based on analysis of GFP expression using the automated fluorescence imaging system (Fig. 5b). The data of mean fluorescence intensity values of the organs isolated from the tumor xenograft mice could demonstrate the tumor metastasis in the mice indirectly ${ }^{20}$. However, the sum fluorescence intensity values for liver, spleen, lung and kidney indicated no significant difference between the sh-LXR $\alpha$ and NC groups (Fig. S3). H\&E staining of the organs was performed, and the results demonstrated that tumor metastases were primarily distributed in the liver (Fig. 5c) (Fig. S4). Vimentin is a useful immunohistochemical marker in ccRCC that can be used to assist in distinguishing ccRCC metastatic tumors ${ }^{21,22}$. IHC of vimentin in this study also revealed that metastatic neoplasm numbers in the liver of LXR $\alpha$ knockdown groups were decreased compared to NC groups (Fig. 5d). In order to further confirm the results, liver metastases mouse model were established through intrasplenical injection. Similar to tail vein injection model, the degree of liver metastases in NC group was higher than that in sh-LXR $\alpha$ group according to the results of mouse weight, macroscopic metastases on the surface of the liver and automated fluorescence imaging system (Figs. 6a-c). Meanwhile, the $\mathrm{H} \& \mathrm{E}$ staining and IHC stain (vimentin) of livers were performed and demonstrated the same results (Figs. 6d, e). According to our results, we demonstrated that LXR $\alpha$ functions as an important oncogene and could modulate the metastasis of renal cancer cells by down-regulate the NLRP3 inflammasome (Fig. 6f).

\section{Discussion}

As a cancer characterized by high mortality and metastasis rates, nearly $35 \%$ of RCC patients who have distance metastases or local relapse after nephrectomy 


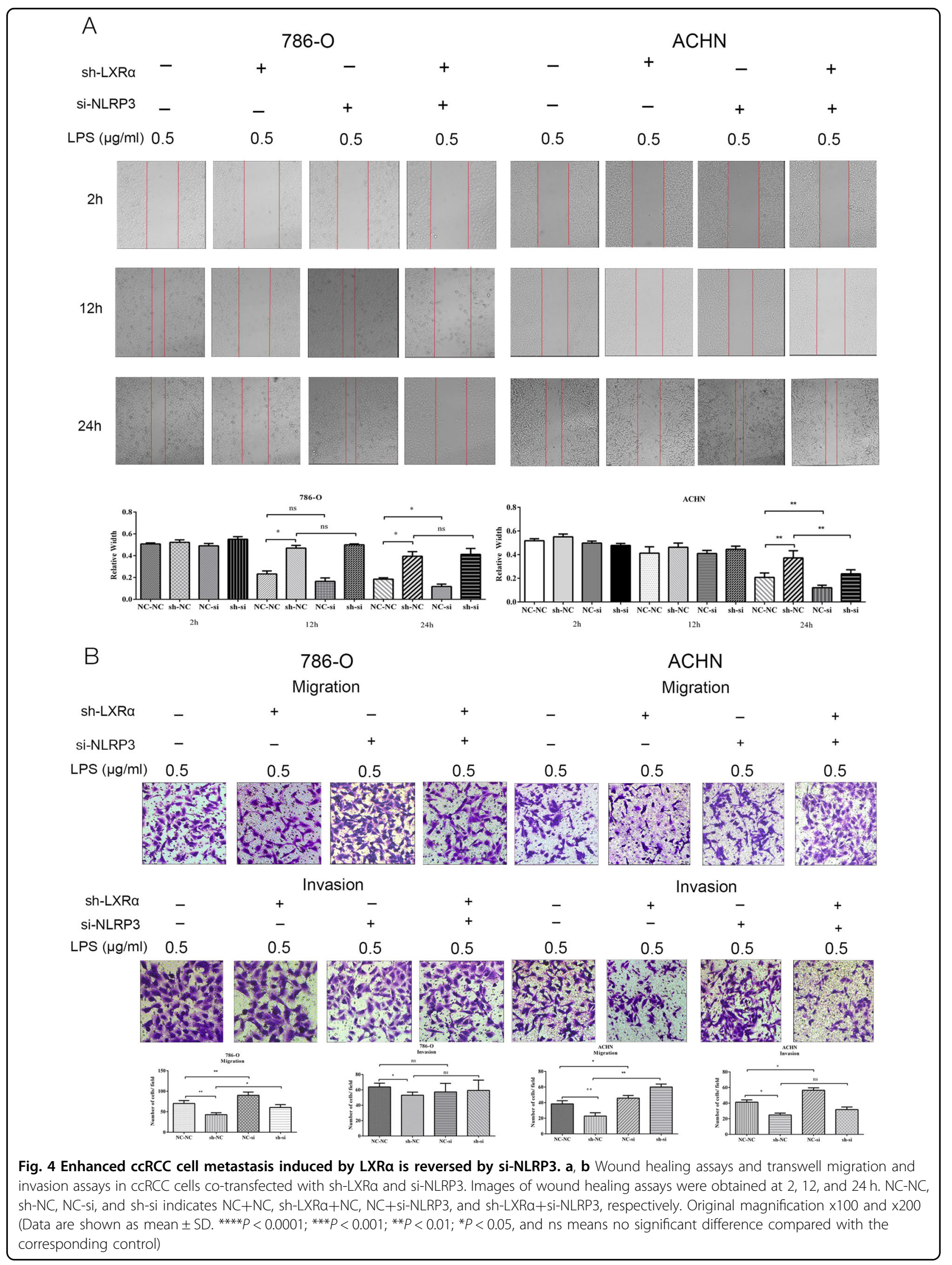




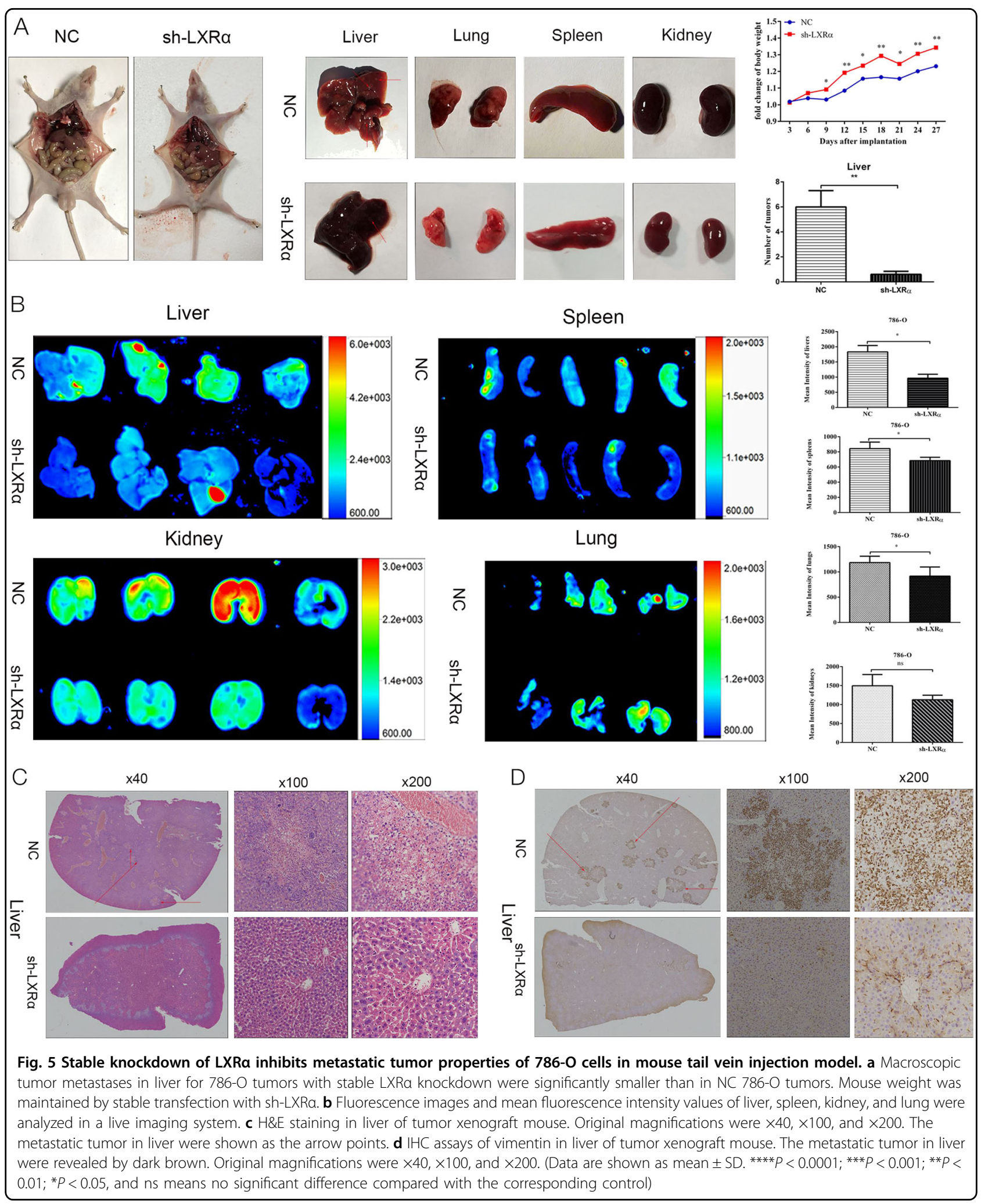

need further chemotherapy or radiotherapy. Unfortunately, RCC is not sensitive to the majority of current chemotherapy and radiotherapy modalities for renal cancer ${ }^{4}$. The management of RCC, especially advanced RCC patients, requires novel and significant findings on the molecular mechanisms regulating tumorigenesis 


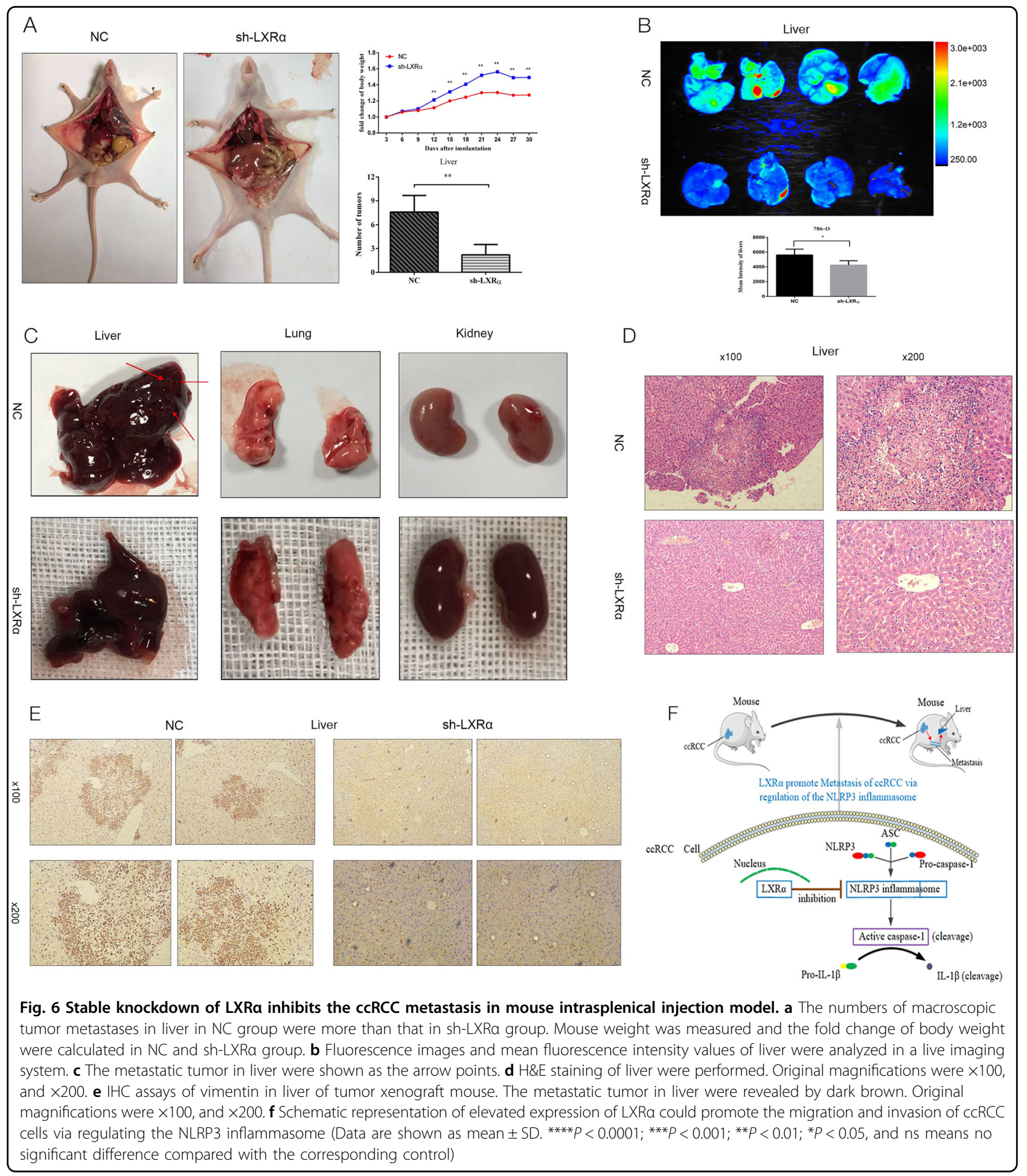

and metastasis of RCC to explore druggable biomarkers and therapeutic targets. According to the studies, several biomarkers including CAIX, SAA and CXCR4 have been proved to be prognostic biomarkers which are related to stage and aggressiveness of tumors ${ }^{23-25}$. However, the majority of these biomarkers are related to only some of the pathological features, diagnosis and prognosis. Thus, reliable biomarkers for diagnosis and prognostic assessment and therapeutic targets in RCC are urgently needed.

As we mentioned before, LXRs have been shown to serve as important regulators in several cancers, including 
breast cancer, prostate cancer. Activation of LXRs could inhibit the proliferation and invasion of prostate cancer via the beta-catenin pathway ${ }^{26}$. GW3965, a LXR agonist, dampen the cell proliferation in human T-cell acute lymphoblastic leukemia cells ${ }^{27}$. In lung cancer, LXR $\alpha$ is demonstrated to be an independent prognostic biomarker indicating a better survival ${ }^{28}$. Although the LXR agonists have been researched as anti-cancer drugs, SR9243 which is a LXR inverse agonist could inhibit the transcriptional activity of LXRs is proved to disrupt cancer cell growth ${ }^{29}$. The role of LXRs in RCC has not been reported up to now. According to our bioinformatics analysis results, LXR $\alpha$ or LXR $\beta$ expression is elevated in ccRCC indicated a poor prognosis. However, considering the degree of expression difference of LXR $\alpha$ or LXR $\beta$ in ccRCC cancer tissues and normal kidney tissues as well as the tissue pattern expression difference of LXR $\alpha$ and $\operatorname{LXR} \beta$, we decided to focus on the expression and biological function of LXR $\alpha$ in ccRCC for the remainder of the study. Intriguingly, our results demonstrate that LXR $\alpha$ might function as an oncogene that could serve as a novel diagnostic and prognostic biomarker in ccRCC for the first time. Additionally, bioinformatics analysis of LXR $\beta$ in ccRCC as well as qRT-PCR results of LXR $\beta$ in ccRCC indicated that LXR $\beta$ might also be an oncogene in ccRCC. The differences in expression patterns between LXR $\alpha$ and LXR $\beta$ and their biological function in ccRCC will be a part of our future research efforts.

According to the studies, a vital role of LXRs is modulating effects of inflammatory and immune responses via transrepression, influencing macrophage polarization and regulating fatty acid synthesis ${ }^{30-32}$. Most reports have focused on the regulatory effects of LXRs on inflammatory or immune responses in the tumor microenvironment or immune cells, including macrophages, regulatory $\mathrm{T}$ cells and monocytes ${ }^{10,33}$. The regulation by LXRs of tumorigenesis or metastasis in cancer via directly affecting inflammation has been rarely reported prior to this study. The inflammasome, a novel inflammatory response complex, has been a hot area for research in recent years. Based on studies revealing a correlation between LXRs and inflammation induced by LPS, we hypothesized that the NLRP3 inflammasome might be a potential target of LXR $\alpha$ in RCC $^{34}$. Although increasing studies addressing the roles of the inflammasome in the development of cancers have been performed over the past few years, the function of the inflammasome in cancer is still uncertain. Several studies indicate that activation of inflammasomes is a protective factor against tumorigenesis, while other reports reach the opposing conclusion ${ }^{35,36}$. The role of the inflammasome in renal cancer has not been reported yet. In our study, we demonstrated that expression levels of NLRP3 in ccRCC cancer tissue were significantly lower than in normal kidney tissue. In addition, we revealed that the NLRP3 inflammasome might function as a tumor suppressor in ccRCC according to the wound healing, transwell migration and invasion assays. Furthermore, the activation of inflammasome could impact the tumor microenvironment, the anti-tumor immune responses and angiogenesis via releasing of the inflammatory factors and chemokines ${ }^{37,38}$. In our study, we found that the function of LXR $\alpha$ that was performed through the NLRP3 inflammasome was mainly focus on the metastasis in ccRCC. Thus, we conjecture that the released cytokines from the cells with active infammasome could influence the metastasis of ccRCC cells through regulation of tumor immunity or angiogenesis in ccRCC. Exploring the downstream pathways of the NLRP3 inflammasome would be another research orientation for us in future.

\section{Conclusions}

According to our study, we found that LXR $\alpha$ was upregulated and related with a poor prognosis in ccRCC. Besides, LXR $\alpha$ could promote the tumor metastasis by down-regulating the NLRP3 inflammasome in ccRCC. The expression of NLRP3 inflammasome was upregulated in renal cell carcinoma compared with the normal kidney tissue. The NLRP3 inflammasome might be a tumor suppressor in RCC. According to our research, we considered that the LXR $\alpha$ and NLRP3 inflammasome had the possibility to be novel therapeutic targets in renal cell cancer.

\section{Materials and methods \\ Bioinformatics analysis}

Using the TCGA database, we obtained the standardized expression levels of LXR mRNA in renal cancer and their correlation to clinical features (age, gender, G stage, TNM stage, overall survival (OS), etc.). Additionally, we used the Oncomine database, including the Gumz Renal dataset, Jones Renal dataset, Yusenko Renal dataset, Lenburg Renal dataset and Beroukhim Renal dataset to assess LXR mRNA data.

\section{Human ccRCC clinical specimens}

A total of $60 \mathrm{ccRCC}$ patient samples were obtained from Wuhan Union Hospital between 2014 and 2017. The samples included both cancer tissue and adjacent normal tissue ( $1 \mathrm{~cm}$ away from the margin of the tumor tissues). We used these tissue samples to perform western blotting, quantitative real-time PCR (qRT-PCR) and IHC. All patients included in the study had no history of adjuvant therapy (chemotherapy or radiotherapy), and informed consent was obtained prior to surgery. The use of these samples was ratified by the Ethics Committee of Human Research of Huazhong University of Science and Technology (HUST). 
Cell culture, lentivirus infection, and transient transfection assay

ccRCC cell lines, including 786-O, ACHN, A498, OSRC-2, Caki-1 and a proximal tubular cell (PTC) line, HK-2, which is derived from normal kidney, were purchased from the American Type Culture Collection (ATCC) in the USA. High-glucose DMEM containing $10 \%$ FBS and $1 \%$ streptomycin-penicillin (Google Biotechnology, Wuhan, China) was used for cell culture, and cells were maintained in a $37^{\circ} \mathrm{C}$ and $5 \% \mathrm{CO} 2$ culture environment. Lentivirus containing pLent-LXR $\alpha$-GFPPuro or empty vector (Vector) pLent-GFP-Puro (Vigene Biology, Shandong, China) was used to infect ACHN or A498 cells with enhanced infection solution (EIS) (Vigene Biology, Shandong, China) according to the manufacturer's protocol. Similarly, pLent-GFP-sh-LXR $\alpha$-Puro lentivirus or its negative control (NC) pLent-GFP-Puro lentivirus (Vigene Biology, Shandong, China) was used to infect 786-O or ACHN cells. Short-interfering RNA (siRNA) against NLRP3 (si-NLRP3) and its negative control (si-NC) were synthesized by RiboBio (RiboBio, Guangzhou, China) and transfected into ccRCC cells using Lipofectamine 2000 reagents (Invitrogen) according to the manufacturer's instructions.

\section{Inflammasome activation}

After being stimulated by lipopolysaccharide (LPS) $(0.5 \mu \mathrm{g} / \mathrm{ml})$ for $4 \mathrm{~h}$ in DMEM complete medium, cells were incubated with ATP $(5 \mathrm{mM})$ for $1 \mathrm{~h}$ in DMEM complete medium to active the intracellular inflammasome. Afterward, cells or cell supernatants were collected for molecular biology experiments.

\section{Quantitative reverse trancription-PCR}

Total RNA was extracted from ccRCC tissues samples using Trizol Reagent BD (Invitrogen, Carlsbad, CA), and the Nanodrop 2000c spectrophotometer (Thermo Scientific) was used to determine the concentration and purity of RNA after extraction. Then, the RevertAid First Strand cDNA Synthesis Kit (Thermo Scientific, Massachusetts, USA) and reverse transcript primers (RiboBio Biology, Guangzhou, China) were used for reverse transcription of RNA. Quantitative real-time PCR (qRT-PCR) was performed with SYBR Green qPCR Mix (Invitrogen, Carlsbad, CA) using synthesized primers from TSINGKE (TSINGKE, Beijing, China). The Roche Light Cycler system (Roche, Mannheim, Germany) was used for qRT-PCR assays. The primer sequences are as follows:

GAPDH forward: 5'-CCTTCATTGACCTCAACTACA-3'; GAPDH reverse: 5'-GCTCCTGGAAGATGGTGAT-3'; LXR $\alpha$ forward: 5'-TTGCCTTGCTCATTGCT-3';

LXR $\alpha$ reverse: 5'-CATCCGTGGGAACATCA-3'; LXR $\beta$ forward: 5'-CTGCTCATCGCCATCAA-3'; LXR $\beta$ reverse: 5'-CAGGCTCACCAGCTTCAT-3'.

\section{Western blotting}

Briefly, protein from tissues and cells was isolated using RIPA buffer (Beyotime Institute of Biotechnology), and protein concentrations were measured with a BCA kit (Roche Molecular Biochemicals, Mannheim, Germany). Equal amounts of protein were subjected to 10\% SDSPAGE and transferred to PVDF membranes (Millipore, Bedford, USA). PVDF membranes were then immersed in $5 \%$ nonfat dried-milk for membrane blocking and incubated with primary antibodies against LXR $\alpha$, NLRP3 (Sanying, Wuhan, China), caspase-1, IL-1 $\beta$ (Sanying, Wuhan, China), $\alpha$-Tubulin (Cell Signaling Technology), $\beta$-Actin, GAPDH (Santa Cruz Biotechnology)overnight at $4^{\circ} \mathrm{C}$. Next, blots were stained with appropriate secondary antibodies, goat anti-mouse or goat anti-rabbit (Santa Cruz Biotechnology) for $1.5-2 \mathrm{~h}$ at room temperature. Finally, bands were visualized with the ChemiDoc-XRS+ system (Bio-Rad, USA).

\section{Enzyme linked immunosorbent assay (ELISA) analysis}

Cells were counted and plated into 6-well tissue culture plates in equal numbers to achieve $90 \%$ confluence. IL-1 $\beta$ ELISA kit (Sanying, Wuhan, China) was used to measure the concentration of IL- $1 \beta$ in the cellular supernatant according to the manufacturer's protocol.

\section{Immunohistochemistry (IHC) and hematoxylin-eosin (H\&E) staining}

As previously described, tissue samples collected from ccRCC patients and tumor xenograft mice were made into paraffin blocks using the following steps: formalin fixation, dehydration and paraffin embedding. Then, IHC was performed using antibodies against LXR $\alpha$ (Sanying, Wuhan, China) and vimentin (Abclonal, Wuhan, China). A Hematoxylin and Eosin Staining Kit (Beyotime) was used for H\&E staining according the manufacturer's protocol.

\section{Wound healing, transwell migration, and invasion assays}

After counting, cells were plated in equal numbers in 6-well culture plates. Vertical wounds were created with $0.1 \mu \mathrm{l}$ pipette tips, cell confluence was approximately $95 \%$, and the images were acquired at 2, 12, and $24 \mathrm{~h}$. According to the manufacturer's protocol, 24-well transwell chambers (Corning) were used for transwell migration and invasion assays. A total of $1 \times 10^{4}$ cells were added into the upper chamber in $200 \mu \mathrm{l}$ of serumfree medium for the migration assay, while $2 \times 10^{4}$ cells were seeded into the top chamber, which was pre-coated with Matrigel (BD, Franklin Lakes, USA) for the invasion assay. Complete medium with 10\% FBS was added to the bottom chamber to serve as a chemoattractant to stimulate migration or invasion of cells. After $24 \mathrm{~h}$ of incubation, cells on the upper surface of the chamber 
membrane were gently scraped with cotton swabs, and transmigrated cells were fixed with $100 \%$ methanol and stained with $0.1 \%$ crystal violet. Positively stained cells were photographed and counted in five random fields.

\section{Apoptosis measurement and cell cycle assays}

Apoptosis assays were performed with the Annexin VFITC/propidium iodide (PI) apoptosis detection kit from KeyGen Biotech according to manufacturer's instructions. FACS (BD Biosciences) and ModFit LT software were used to detect and analyze the percentage of apoptotic cells. After being starved and synchronized in serum-free medium for $24 \mathrm{~h}$, cells were collected and fixed in $75 \%$ ethanol overnight. Cells were subsequently incubated with PI and RNase A for $30 \mathrm{~min}$ at $37^{\circ} \mathrm{C}$ in the dark. The percentage of cells in each cell cycle (G0/G1, S, G2/M stage) was also detected and analyzed using FACS (BD Biosciences) and ModFit LT software.

\section{Tumor xenograft model}

786-O cells stably transfected with sh-LXR $\alpha$ lentivirus or negative control lentivirus were injected into 3-4-weekold BALB/C nude mice (HFK Bio-technology, Beijing, China) by tail vein injection and intrasplenical injection. Each group contained five mice. Before injection, the inflammasome in 786-O cells (NC and sh-LXR $\alpha$ group) were actived through the methods mention above. The mice were weighed every three days after injection. In addition, a live imaging system was used for observation of metastatic tumor lesions. Organs excised from mice were paraffin-embedded for IHC and H\&E staining. IHC assays of organs were performed using the antibody against vimentin (Abclonal, Wuhan, China) as described above. The animal experiments were authorized by the Animal Ethics Committee of HUST.

\section{Statistical analysis}

In this study, Graphpad Prism 6.0 (GraphPad Software, San Diego, California, USA) and SPSS 22.0 (IBM, New York, NY) statistical software were used. T-test was used for analysis of the significant differences in LXRs mRNA expression in normal kidney tissue and matched renal cancer tissue. Mann-Whitney test, Pearson's Chi-square test and a receiver operator characteristic curve were used for analysis of LXR expression in ccRCC subgroups, correlations between LXR expression and clinicopathological features of ccRCC, and the diagnostic values of LXR in ccRCC patients. Relationships between LXR mRNA expression and overall survival (OS) rate or disease-free survival rate were analyzed using the log-rank test and Kaplan-Meier curves. Results of $P<0.05$ were considered significant.
Data availability

You could find all data that is used for evaluating the conclusions in the paper and the Supplementary materials. Other related data of this study could be requested from the authors.

\section{Acknowledgements \\ The authors thank the support provided by National Natural Science Foundation of China (Grant No. 81272560, 81672524 \& 81672528) and the Program for New Century Excellent Talents in University from the Department of Education of China (NCET-08-0223). This study was supported by the National Natural Science Foundation of China (Grant No. 81672524 \& 81672528) and the Program for New Century Excellent Talents in University from the Department of Education of China (NCET-08-0223).}

\section{Author details \\ ${ }^{1}$ Department of Urology, Union Hospital, Tongji Medical College, Huazhong University of Science and Technology, Wuhan 430022, China. ${ }^{2}$ Institute of Urology, Union Hospital, Tongji Medical College, Huazhong University of Science and Technology, Wuhan 430022, China. ${ }^{3}$ Department of Pathogenic Biology, School of Basic Medicine, Huazhong University of Science and Technology, Wuhan 430030, China}

\section{Ethics approval and consent to participate}

This study was approved by Ethics Committee of Huazhong University of science and technology (HUST) and all study participants signed an informed consent.

Conflict of interest

The authors declare that they have no conflict of interest.

\section{Publisher's note}

Springer Nature remains neutral with regard to jurisdictional claims in published maps and institutional affiliations.

Supplementary Information accompanies this paper at (https://doi.org/ 10.1038/s41419-019-1345-3).

Received: 9 September 2018 Revised: 6 January 2019 Accepted: 7 January 2019

Published online: 15 February 2019

\section{References}

1. Siegel, R. L., Miller, K. D. \& Jemal, A. Cancer statistics, 2018. CA. 68, 7 (2018).

2. Linehan, W. M. Genetic basis of kidney cancer: role of genomics for the development of disease-based therapeutics. Genome Res 22, 2089 (2012).

3. Gong, J., Maia, M. C., Dizman, N., Govindarajan, A. \& Pal, S. K. Metastasis in renal cell carcinoma: Biology and implications for therapy. Asian J. Urol. 3, 286 (2016).

4. Choueiri, T. K. Clinical treatment decisions for advanced renal cell cancer. J. Natl. Compr. Canc Netw. 11, 694 (2013).

5. Hong, C. \& Tontonoz, P. Coordination of inflammation and metabolism by PPAR and LXR nuclear receptors. Curr Opin Genet DeV 18, 461 (2008).

6. Bobin-Dubigeon, C. et al. Liver X Receptor (LXR)-regulated Genes of Cholesterol Trafficking and Breast Cancer Severity. Anticancer Res 37, 5495 (2017).

7. Davalieva, K. et al. Comparative proteomics analysis of urine reveals downregulation of acute phase response signaling and LXR/RXR activation pathways in prostate cancer. Proteomes 6, pii: proteomes6010001 (2017).

8. Goodwin, B. J., Zuercher, W. J. \& Collins, J. L. Recent advances in liver X receptor biology and chemistry. Curr Top Med Chem 8, 781 (2008).

9. Pascual-Garcia, M. et al. Reciprocal negative cross-talk between liver $X$ receptors (LXRS) and STAT1: effects on IFN-gamma-induced inflammatory responses and LXR-dependent gene expression. J. Immunol 190, 6520 (2013).

10. Pourcet, B. et al. The nuclear receptor LXR modulates interleukin-18 levels in macrophages through multiple mechanisms. Sci. Rep. 6, 25481 (2016). 
11. Morello, F. et al. LXR-activating oxysterols induce the expression of inflammatory markers in endothelial cells through LXR-independent mechanisms. Atherosclerosis 207, 38 (2009).

12. Dunn, J. H., Ellis, L. Z. \& Fujita, M. Inflammasomes as molecular mediators of inflammation and cancer: potential role in melanoma. Cancer Lett 314, 24 (2012).

13. Guo, B., Fu, S., Zhang, J., Liu, B. \& Li, Z. Targeting inflammasome/lL-1 pathways for cancer immunotherapy. Sci. Rep. 6, 36107 (2016).

14. Davis, B. K., Wen, H. \& Ting, J. P. The inflammasome NLRs in immunity, inflammation, and associated diseases. Annu Rev Immunol 29, 707 (2011).

15. Du, Q. et al. Dietary cholesterol promotes AOM-induced colorectal cancer through activating the NLRP3 inflammasome. Biochem Pharmacol 105, 42 (2016).

16. Wang, Y. et al. Activation of NLRP3 inflammasome enhances the proliferation and migration of A549 lung cancer cells. Oncol Rep 35, 2053 (2016).

17. $\mathrm{Hu}, \mathrm{H}$. et al. Long non-coding RNA XLOC_000647 suppresses progression of pancreatic cancer and decreases epithelial-mesenchymal transition-induced cell invasion by down-regulating NLRP3. Mol Cancer 17, 18 (2018).

18. Huang, C. F. et al. NLRP3 inflammasome activation promotes inflammationinduced carcinogenesis in head and neck squamous cell carcinoma. J. Exp. Clin. Cancer Res 36, 116 (2017).

19. Sharma, D. \& Kanneganti, T. D. The cell biology of inflammasomes: Mechanisms of inflammasome activation and regulation. J. Cell Bio/ 213, 617 (2016).

20. Chen, $X$. et al. miR-141 is a key regulator of renal cell carcinoma proliferation and metastasis by controlling EphA2 expression. Clin Cancer Res 20, 2617 (2014).

21. von Brandenstein, M. et al. Vimentin 3, the new hope, differentiating RCC versus oncocytoma. Dis Markers 2015, 368534 (2015).

22. Zhao, W. et al. DOG1, cyclin D1, CK7, CD117 and vimentin are useful immunohistochemical markers in distinguishing chromophobe renal cell carcinoma from clear cell renal cell carcinoma and renal oncocytoma. Pathol Res Pract 211, 303 (2015).

23. Choueiri, T. K. et al. Carbonic anhydrase IX as a potential biomarker of efficacy in metastatic clear-cell renal cell carcinoma patients receiving sorafenib or placebo: analysis from the treatment approaches in renal cancer global evaluation trial (TARGET). Urol. Oncol. 31, 1788 (2013).
24. Fischer, K., Theil, G., Hoda, R. \& Fornara, P. Serum amyloid A: a biomarker for renal cancer. Anticancer Res 32, 1801 (2012).

25. Rasti, A. et al. Reduced expression of CXCR4, a novel renal cancer stem cell marker, is associated with high-grade renal cell carcinoma. J. Cancer Res Clin. Oncol. 143, 95 (2017).

26. Youlin, $\mathrm{K}$. et al. Liver $\mathrm{X}$ receptor activation inhibits $\mathrm{PC}-3$ prostate cancer cells via the beta-catenin pathway. Pathol Res Pract 213, 267 (2017).

27. Zhang, R., Liu, Z., Li, Y. \& Wu, B. LXR agonist regulates the proliferation and apoptosis of human T-Cell acute lymphoblastic leukemia cells via the SOCS3 pathway. Int J. Biochem Cell Biol. 78, 180 (2016).

28. Melloni, $\mathrm{G}$. et al. Prognostic role of liver $\mathrm{X}$ receptor-alpha in resected stage ॥ and III non-small-cell lung cancer. Clin Respir J. 12, 241 (2018).

29. Flaveny, C. A. et al. Broad anti-tumor activity of a small molecule that selectively targets the warburg effect and lipogenesis. Cancer Cell 28, 42 (2015).

30. Li, P. et al. NCoR repression of LXRs restricts macrophage biosynthesis of insulin-sensitizing omega 3 fatty acids. Cell 155, 200 (2013).

31. Marathe, $C$. et al. Preserved glucose tolerance in high-fat-fed C57BL/6 mice transplanted with PPARgamma-/-, PPARdelta-/-, PPARgammadelta-/-, or LXRalphabeta-/- bone marrow. J. Lipid Res 50, 214 (2009).

32. Gillespie, M. A. et al. An LXR-NCOA5 gene regulatory complex directs inflammatory crosstalk-dependent repression of macrophage cholesterol efflux. EMBO J. 34, 1244 (2015).

33. Kim, B. Y. et al. 27-Hydroxycholesterol upregulates the production of heat shock protein 60 of monocytic cells. J. Steroid Biochem Mol. Biol. 172, 29 (2017).

34. $Y u, S . X$. et al. Liver $X$ receptors agonists suppress NLRP3 inflammasome activation. Cytokine 91, 30 (2017).

35. Allen, I. C. et al. The NLRP3 inflammasome functions as a negative regulator of tumorigenesis during colitis-associated cancer. J. Exp. Med. 207, 1045 (2010).

36. Gasparoto, T. H. et al. Inflammasome activation is critical to the protective immune response during chemically induced squamous cell carcinoma. PLOS ONE 9, e107170 (2014).

37. van Deventer, H. W. et al. The inflammasome component NLRP3 impairs antitumor vaccine by enhancing the accumulation of tumor-associated myeloid-derived suppressor cells. Cancer Res 70, 10161 (2010).

38. Voronov, E. et al. Unique versus redundant functions of IL-1alpha and IL-1beta in the tumor microenvironment. Front Immunol 4, 177 (2013). 